\title{
Correlates of nonmedical use of stimulants and methamphetamine use in a national sample
}

\author{
Lian-Yu Chen, M.D. ${ }^{a, d}$, Eric C. Strain, M.D. ${ }^{b}$ Pierre Kébreau Alexandre, PhD., MS, MPH. ${ }^{a}$, G. \\ Caleb Alexander, M.D., MPH ${ }^{c, d}$, Ramin Mojtabai, M.D., MPH, PhD. ${ }^{a, b, d}$, and Silvia S. Martins, \\ M.D., PhD. ${ }^{a, e}$ \\ aDepartment of Mental Health, Johns Hopkins Bloomberg School of Public Health, $624 \mathrm{~N}$. \\ Broadway, $7^{\text {th }}$ floor, Baltimore, MD 21205 \\ bDepartment of Psychiatry, Johns Hopkins School of Medicine, 5510 Nathan Shock Drive \\ Baltimore, MD 21224 \\ 'Department of Epidemiology, Johns Hopkins Bloomberg School of Public Health, 615 N. Wolfe \\ Street W6035, Baltimore, MD 21205 \\ ${ }^{\mathrm{d} C e n t e r}$ for Drug Safety and Effectiveness, Johns Hopkins University, 615 N. Wolfe Street \\ W6035, Baltimore, MD 21205 \\ eDepartment of Epidemiology, Mailman School of Public Health, Columbia University, 722 West \\ 168th street, Rm. 509, New York, NY 10032
}

\begin{abstract}
Background-Despite chemical similarities, ADHD stimulants and methamphetamine have distinct use patterns in the community. This study compared the characteristics of nonmedical ADHD stimulants users and methamphetamine users in a household sample.
\end{abstract}

\footnotetext{
(C) 2014 Elsevier Ltd. All rights reserved.

Correspondence to: Lian-Yu Chen, M.D., Johns Hopkins Bloomberg School of Public Health, Mental Health Department, 624 N. Broadway, Suite 784, Baltimore, MD 21205-1900, United States, Tel: 410-929-9303, Fax: 410-955-9088, liachen@jhsph.edu. Conflict of Interest Disclosures:

Dr. Mojtabai has received consulting fees from Lundbeck Pharmaceuticals. Dr. Strain used to receive consulting fee from Shire Pharmaceuticals. Dr. Alexander is an ad hoc member of the FDA's Drug Safety and Risk Management Advisory Committee, serves as a paid consultant to IMS Health and serves on an IMS Health scientific advisory board (this arrangement has been reviewed and approved by Johns Hopkins University in accordance with its conflict of interest policies). Other authors declare they have no conflicts of interest.

Author Contributions:

Study concept and design: Lian-Yu Chen, Eric C. Strain, Silvia S. Martins, Ramin Mojtabai

Acquisition of data: The data reported herein come from the 2009-2011 National Survey of Drug Use and Health (NSDUH) public data files available at the Substance Abuse and Mental Health Data Archive and the Inter-university Consortium for Political and Social Research, which are sponsored by the Office of Applied Studies, Substance Abuse and Mental Health Services Administration. Analysis and interpretation of data: Lian-Yu Chen, Ramin Mojtabai \& Silvia S. Martins

Critical revision of the manuscript for important intellectual content: Drs. Silvia S. Martins, G. Caleb Alexander, Eric C. Strain, Ramin Mojtabai \& Pierre Kébreau Alexandre

Statistical analysis: Lian-Yu Chen

Publisher's Disclaimer: This is a PDF file of an unedited manuscript that has been accepted for publication. As a service to our customers we are providing this early version of the manuscript. The manuscript will undergo copyediting, typesetting, and review of the resulting proof before it is published in its final citable form. Please note that during the production process errors may be discovered which could affect the content, and all legal disclaimers that apply to the journal pertain.
} 
Methods-In data from the 2009-2011 National Survey on Drug Use and Health, adult and adolescent stimulant users were categorized into three mutually exclusive subgroups: nonmedical ADHD stimulant users only (STM users), methamphetamine users (METH users), and both nonmedical ADHD stimulant and methamphetamine users (STM/METH users). Multivariate logistic regression analyses identified the substance comorbidity, mental health, and deviant behavior characteristics associated with these three groups.

Results-Compared to adolescent STM users, STM/METH users were more likely to be female, younger and uninsured while METH users were more likely to be younger, in a minority group and from a higher-income family. Compared to adult STM users, METH and STM/METH users were more likely to be male, older, uninsured, no longer married, and to be from rural areas. Adolescent METH users were more likely than STM users to report illegal drug use while adult METH users were less likely to report prescription drug use than their STM user counterparts. Overall, adult and adolescent STM/METH users were more likely to report substance use, mental health problems and deviant behaviors compared to STM users.

Conclusion-The characteristics of STM users differ from METH and STM/METH users, and their associations with substance use and psychiatric comorbidities differ by age. Findings have implications for understanding the risks for stimulant use in different age subgroups.

\section{Keywords}

Prescription stimulants; Substance abuse; Methamphetamine use; Mental health; Deviant behaviors

\section{Introduction}

The past two decades have witnessed increased public concerns and research regarding nonmedical use of prescription stimulants (Einhorn et al., 2012), particularly those commonly prescribed for attention-deficit hyperactivity disorder (ADHD) (McCabe et al., 2005, McCabe \& Teter, 2007, McCabe et al., 2004, Safer et al., 1996, Teter et al., 2005, Teter et al., 2006). Previous studies provide strong evidence that nonmedical use of prescription stimulants is a growing problem especially among youth (Arria et al., 2008, Johnston, 2003, McCabe et al., 2005, SAMHSA, 2009a, Teter et al., 2006, White et al., 2006), and is associated with other substance use, mental health comorbidities, criminal involvement, and cardiovascular conditions (NIDA, 2009).

Studies based on Monitoring the Future (MTF) and the National Survey on Drug Use and Health (NSDUH) both showed that college students were twice more likely to report nonmedical use of prescription stimulants than their counterparts not attending college (Johnston, 2003, SAMHSA, 2009a). There are also reports that adolescents increasingly use prescription stimulants nonmedically. In a web survey of a secondary school, $17.5 \%$ of students reported both medical and nonmedical use of prescription stimulants, and 3.3\% reported nonmedical use only (McCabe \& Teter, 2007). Another study of middle and high schools showed prescription stimulants were used by $4.5 \%$ of students, with $23.3 \%$ reporting being approached to sell, give, or trade these drugs (McCabe et al., 2004). The high prevalence of nonmedical use among students could be explained by the use of these drugs for cognitive enhancement. In past research, the most commonly reported motives for 
nonmedical prescription stimulants use in this age group were to help with concentration or to study, followed by recreational use (Teter, et al., 2005; Teter, et al., 2006).

Methamphetamine ("speed," "ice," "crystal," “crank," or "glass") is a highly addictive stimulant which is similar in structure to amphetamine (DEA, 2013, Wu et al., 2007). Although methamphetamine can be legitimately prescribed for treatment of ADHD, narcolepsy and obesity, the medical use of this substance is extremely limited (NIDA, 2009). Most of methamphetamine use is illegal and has been linked to other substance use disorders (DEA, 2013, Furr et al., 2000), high-risk sexual behaviors (Frosch et al., 1996), HIV and HCV infections (Frosch et al., 1996, Gonzales et al., 2006), psychiatric and behavior problems (Brecht et al., 2004, Gruenewald et al., 2010, Zweben et al., 2004), and serious physical conditions (Darke et al., 2008, Kaye et al., 2008, Yu et al., 2003). In a recent survey, about 11.9 million Americans aged 12 and older reported lifetime use of methamphetamine (SAMHSA, 2012). Methamphetamine was reported as the primary substance of abuse in $8 \%$ of all substance use treatment admissions, and accounted for $99 \%$ of non-cocaine stimulant admissions (SAMHSA, 2009b).

ADHD stimulants and methamphetamine share several similarities. First, these drugs are classified as schedule II based on the Controlled Substances Act (CSA), indicating their high abuse potential (DEA, 2003). Second, nonmedical use of these drugs occurs mostly in young individuals who are more likely to engage in other substance use or deviant behaviors (Brecht et al., 2004, McCabe et al., 2005). Finally, ADHD stimulants and methamphetamine are central nervous system stimulants with similar physiological effects such as activating catecholamine system and adverse cardiovascular effects (Findling et al., 2001, Newton et al., 2005). Despite these similarities, ADHD stimulants are distinct from methamphetamine in that they are legitimately prescribed drugs, while the source of methamphetamine is mainly illegal. More than $70 \%$ of nonmedical ADHD stimulant users reported their source to be a friend who had legitimately obtained a prescription (Garnier-Dykstra et al., 2012). In contrast, illegal production in clandestine laboratories using diverted pseudoephedrine products and Mexican drug trafficking organizations contributed greatly to its widespread availability (Cody, 2002, DEA, 2013).

Previous studies examining these two types of stimulants focused on young adults only and were conducted prior to 2003 (Herman-Stahl et al., 2006, 2007, Wu et al., 2007).

Methamphetamine use decreased in the mid-2000s and then increased again around 20092010, suggesting the need to conduct analyses using more contemporary data (Maxwell \& Brecht, 2011). Our study builds on earlier studies and further fills the information gap by comparing nonmedical ADHD stimulant users to methamphetamine users on a broad range of characteristics including socio-demographic, mental health and deviant behavior profiles, and other substance use comorbidities (Herman-Stahl et al., 2006, 2007, Wu et al., 2007).

More specifically, in this study we examined the prevalence and correlates of nonmedical use of ADHD stimulant, methamphetamine use, and both nonmedical ADHD stimulant and methamphetamine use among adults (aged 18 and above) and adolescents (aged 12-17) using data from the 2009-2011 NSDUH. We hypothesized that (1) the three groups would differ with regard to important socio-demographic characteristics such as gender and age; 
and (2) methamphetamine use alone and in conjunction with nonmedical ADHD stimulant use would be more strongly associated with comorbid mental health conditions, deviant behaviors and other substance use than nonmedical ADHD stimulant use.

\section{Methods}

\subsection{Study sample and measures}

The NSDUH is an annual cross-sectional survey sponsored by the Substance Abuse and Mental Health Administration (SAMHSA) and is designed to provide estimates of the prevalence of alcohol and drug use in the household population of the United States. The survey employs a 50-state design with an independent multistage area probability sample for each of the 50 states and the District of Columbia. We used data from the $2009(n=55,772)$, $2010(n=55,873)$ and $2011(n=58,397)$ NSDUH public-use data files; three consecutive NSDUH years were combined in order to increase sample size. Response rate across the three years ranged from $87 \%$ to $89 \%$ for household screening and from $74 \%$ to $76 \%$ for completed interviews. Survey items were administered by computer-assisted personal interviewing (CAPI) and audio computer-assisted self-interviewing (ACASI). Respondents were offered a $\$ 30$ incentive payment for participation in the survey. There is no overlap of respondents between NSDUH 2009 to 2011. Detailed information about the sampling and survey methodology of NSDUH is found elsewhere (SAMHSA, 2009, 2010, 2011).

\subsubsection{Nonmedical ADHD stimulant and methamphetamine use-ADHD} stimulants, defined as stimulants with specific indication for ADHD treatment, included Ritalin ${ }^{\circledR}$ or methylphenidate, Cylert ${ }^{\circledR}$, Dexedrine ${ }^{\circledR}$, Dextroamphetamine, Adderall $®$, Concerta ${ }^{\circledR}$, and Vyvanse ${ }^{\circledR}$. The survey used separate questions to assess nonmedical use of any ADHD stimulants in the lifetime. Ritalin $®$ or methylphenidate use was assessed in a single question ("Have you ever, even once, used Ritalin or Methylphenidate that was not prescribed for you or that you took only for the experience or feeling it caused?"). A similar question was asked regarding the nonmedical use of Adderall@. However, Cylert@, Dexedrine ${ }^{\circledR}$, dextroamphetamine, Concerta ${ }^{\circledR}$ and Vyvanse $®$ were ascertained by asking respondents to look at all the categories of stimulants shown on a pill card and to identify which stimulants they had ever used nonmedically.

Lifetime methamphetamine use was ascertained by two questions in NSDUH. First, the respondents were asked: "Have you ever, even once, used meth, Desoxyn, or Methedrine that was not prescribed for you or that you took only for the experience or feeling it caused?" Second, the respondents were asked: "Methamphetamine, also known as crank, ice, crystal meth, speed, glass, and many other names, is a stimulant that usually comes in crystal or powder forms. It can be smoked, "snorted," swallowed or injected. Have you ever, even once, used methamphetamine?" NSDUH added the latter question in the noncore section of the survey in order to capture information from respondents who may have used methamphetamine but did not recognize it as a prescription drug. Methamphetamine use was defined by a positive response to either question.

Based upon responses to the above questions, we created three mutually exclusive groups of respondents: (1) nonmedical users of ADHD stimulants excluding methamphetamine users 
(STM users); (2) methamphetamine users excluding nonmedical ADHD stimulants users (METH users); and (3) participants who used both ADHD stimulants nonmedically and methamphetamine (STM/METH users).

2.1.2. Socio-demographic measures-Socio-demographic variables included in the analyses for adults were gender, age (18-25, 26-34, 35-49, and >50 years), race/ethnicity (white, black, Hispanic, other), marital status (married or living as married, divorced/ separated/widowed, never married), employment status (partial or full employment, unemployed, not in labor force), education (less than high school, high school, college and above), annual household income ( $\leq \$ 19,999, \$ 20,000-\$ 34,999, \$ 35,000-\$ 69,999$, $\geq$ $\$ 70,000$ ), health insurance status (no insurance, any insurance), and urbanicity (metropolitan, suburban, rural). Socio-demographic variables for adolescents were gender, age (12-13, 14-15, and 16-17 year), race/ethnicity, annual household income, insurance status, and urbanicity.

2.1.3. Other substances use measures-Past-year marijuana, cocaine, heroin, hallucinogen, ecstasy, tranquilizer, sedative, inhalant, alcohol, and prescription opioid use were assessed by asking participants a series of questions worded as follows: "Have you ever, even once, used [alcohol or drug name]?").

2.1.4. Past-year mental health and deviant behavior variables-Past-year mental health treatment use was ascertained by asking participants whether they received any mental health treatment in the past 12 months, including inpatient, outpatient treatment or counseling for emotional or behavioral problems. NSDUH explicitly asked the respondents not to include treatment for alcohol or drug use.

Past-year major depressive episode (MDE) was ascertained using a structured interview based on the Diagnostic and Statistical Manual of Mental Disorders-IV (DSM-IV) criteria (American Psychiatric Association, 1994). The diagnostic assessment was modeled after the Composite International Diagnostic Interview (CIDI) as implemented in the National Comorbidity Survey-Replication (NCS-R) study (Kessler et al., 2003). CIDI has been shown to have an acceptable level of agreement with clinician-administered interviews (Andrews \& Peters, 1998, Wittchen, 1994).

Past-year anxiety disorder was ascertained by asking the respondents if they were told by "a doctor or other medical professional" that they had an "anxiety disorder" in the past 12 months.

Past-year deviant behaviors were assessed by asking the respondents how many times in the past 12 months they had been arrested and booked for breaking the law (not counting minor traffic violations), had attacked someone with the intent to seriously hurt them, had sold illegal drugs, and had stolen or tried to steal anything worth more than $\$ 50$. These four indicators of deviant behaviors were assessed for both adolescent and adult participants. Consistent with past research, participants who reported any of the four behaviors were categorized as having "deviant behavior" ( 0 for none of these behaviors and 1 for engaging in these behaviors 1 time or more) (Martins et al., 2006; Martins et al., 2009). 


\subsection{Statistical analyses}

Analyses were conducted in two stages. First, we compared socio-demographic characteristics of METH users, and STM/METH users, to STM users (the reference group for all analyses). Next, we compared METH and STM/METH users to the STM users on measures of past-year substance use, mental health profile (past-year MDE, anxiety disorder, mental health service use), and deviant behaviors. These comparisons were conducted separately for adults and adolescents (with the age cutoff of 18). Multivariate logistic regression analyses were performed within age groups for adults and adolescents adjusted for age, gender, race, income, insurance, urbanicity, and year. Analyses in adult group were additionally adjusted for marital status, education, and employment status.

Data were weighted to reflect the complex design of the NSDUH sample and were analyzed using Stata 13.0 software (StataCorp, 2013). We used Taylor series linearization method to obtain proper standard error estimates.

\section{Results}

\subsection{Socio-demographic profiles of adolescent and adult users (Tables 1 and 2)}

The prevalence of METH and STM/METH use was higher in adults than in adolescents while STM use had a similar prevalence in the two groups. Among adolescent respondents aged $12-17,2.6 \%(n=1,592)$ were categorized as STM users, $0.4 \%(n=197)$ as METH users and $0.4 \%(\mathrm{n}=229)$ as STM/METH users. Among the adult respondents aged 18 and above, $3.1 \%(\mathrm{n}=7,267)$ were categorized as STM users, $4.5 \%(\mathrm{n}=4,640)$ as METH users and $1.5 \%$ $(\mathrm{n}=2,282)$ as $\mathrm{STM} / \mathrm{METH}$ users.

Compared to STM users, adolescent STM/METH users (Table 1) were more likely to be female, Hispanic, and to reside in a suburban than metropolitan setting, but less likely to be older (aged 16-17 vs. aged 12-13), and insured. Adolescent METH users (vs. STM users) were more likely to be in a minority group (vs. white) and from a higher-income family (household income $\geq \$ 75,000$ vs. $<\$ 20,000$ ) but less likely to be older (aged 16-17 vs. aged 12-13).

For adults (Table 2), the age differences across the three groups were remarkably salient: $50.7 \%$ of the STM users were between age $18-25 ; 72.9 \%$ of METH users and $55.0 \%$ of STM/METH users were older than 35 years. Compared to adult STM users, adult METH and STM/METH users were more likely to be male, older, uninsured, no longer married (vs. currently married or living as married), having education less than high school, from a higher income family (vs. family income $<\$ 20,000$ ), and from rural areas (vs. urban). Adult METH users were more likely than adult STM users to be of Hispanic or other ethnic groups (vs. non-Hispanic-whites), while no racial differences were found between STM/METH users and STM users.

\subsection{Past-year substance use, mental health and deviant behaviors in adolescents (Table 3)}

Adolescent STM/METH users were significantly more likely than STM users to report all types of past year substance use except for alcohol use. In contrast, adolescent METH users were only more likely to report cocaine $(\mathrm{aOR}=2.15[1.14,4.04])$, ecstasy use (aOR=1.19 
$[1.17,3.10])$ and inhalants $(\mathrm{aOR}=1.97[1.15,3.39])$ compared to STM users. Adolescent STM/METH users were also more likely than STM users to report mental health conditions including anxiety disorder $(\mathrm{aOR}=3.42[2.26,5.16])$, having received mental health treatment $(\mathrm{aOR}=2.07$ [1.41, 3.04]), and all types of deviant behaviors (arrested and booked: aOR=2.21 [1.46, 3.34]; sold illegal drugs: $\mathrm{aOR}=3.57$ [2.22,5.73]; stole: $\mathrm{aOR}=2.46$ [1.56, 3.87]; attacked someone: aOR=2.39 [1.61,3.55]). No differences were observed between METH users and STM users regarding mental health and deviant behaviors.

\subsection{Past-year substance use, mental health and deviant behaviors in adults (Table 4)}

Adult METH users were less likely than STM users to report using prescription drugs nonmedically in the past-year, including prescription opioids (aOR=0.64 [0.51, 0.80]), tranquilizers $(\mathrm{aOR}=0.60[0.44,0.82])$, and sedatives $(\mathrm{aOR}=0.35[0.17,0.72])$. In contrast, adult STM/METH users were more likely than STM users to report using all other substances except for alcohol. There were no differences between METH users and STM users with regard to mental health conditions, whereas STM/METH users were more likely than STM users to report a past-year anxiety disorder $(\mathrm{aOR}=1.75[1.21,2.53])$. Of note, there was a trend for STM/METH users to be more likely to report all types of deviant behaviors examined (arrested and booked: aOR=1.59 [1.24, 2.03]; sold illegal drugs: $\mathrm{aOR}=2.33$ [1.76,3.10]; attacked someone: $\mathrm{aOR}=1.38$ [1.02,1.88]), except for stealing, while METH users were more likely to report being booked or arrested ( $\mathrm{aOR}=1.71[1.29,2.27])$ and less likely to report stealing ( $\mathrm{aOR}=0.61[0.38,0.99])$ as compared to STM users.

\section{Discussion}

There were three main findings in this study. First, socio-demographic characteristics of the three stimulant use groups differed, and the pattern of variations was different in the two age subgroups. For instance, adolescent methamphetamine users were more likely to be female while adult methamphetamine users were more likely to be male (compared to nonmedical ADHD stimulant users). Second, adolescent stimulant users showed remarkably distinct substance use comorbidities compared to adult stimulant users: adolescent METH users were more likely to report illegal drug use as compared to adolescent STM users; whereas, adult METH users were more likely to report prescription drug use compared to adult STM users. Third, adult and adolescent STM/METH users in both age groups had a higher prevalence of substance use, mental health conditions and deviant behaviors compared to STM users.

The gender distribution of the three stimulant user groups also differed between adolescents and adults. In adults, more than $60 \%$ of METH and STM/METH users were male; in contrast, more than half of adolescent METH and STM/METH users were female. Past research suggested that males and females were equally likely to use methamphetamine (Durell et al., 2008, Kroutil et al., 2006, SAMHSA, 2005), while other studies have shown that in the younger age group, females are more likely than males to use methamphetamine (Herman-Stahl et al., 2006, 2007, Wu et al., 2007). In a previous study, females were five times more likely than males to use methamphetamine for weight loss, which might explain why female adolescents are more likely than their male counterparts to use methamphetamine (Brecht, et al., 2004). 
The age differences between the three stimulant users groups in adults were striking. More than half of the adult STM users were aged 18-25; whereas, the majority of adult METH and STM/METH users were older than 35 years old. Our findings corroborate past research on the age distribution of ADHD stimulants which are mainly used nonmedically by adolescents and young adults (Herman-Stahl et al., 2006, 2007, McCabe et al., 2005, SAMSHA, 2008, Teter et al., 2006). Previous NSDUH reports in early 2000s showed that methamphetamine users were mostly aged 18-25 (Durell et al., 2008, SAMHSA, 2005). Our study using the most recent NSDUH data points out that a large proportion of methamphetamine users are in the 35 years old and older age group, suggesting the possibility of a cohort effect - that is, previously young users who are now in their middleage continue using this drug as they age.

Consistent with many prior studies, this study showed nonmedical ADHD users were proportionally more likely to be white (McCabe et al., 2007, McCabe et al., 2005, Teter et al., 2006). Our study further revealed that METH users were significantly more likely than STM users to be Hispanic in both the adolescent and adult groups. Since Hispanics now comprise the largest and fastest growing minority group in the US (Ennis, 2011), the fact that METH users (vs. STM users) were more likely to be Hispanics than whites highlights the need for culturally-sensitive preventive strategies targeting Hispanics to diminish methamphetamine use in this subpopulation.

The finding of significant comorbidity with other substances is consistent with past research (Brecht et al., 2004, Durell et al., 2008, McCabe \& Teter, 2007, NIDA, 2009, Teter et al., 2006). One unique finding is that adolescent METH users had different drug use profiles than adult METH users. Adolescent METH users were more likely to have used other stimulants (cocaine and ecstasy) compared to adolescent STM users. In contrast, adult METH users were less likely to report using other prescription drugs nonmedically compared to adult STM users. As the most common source of nonmedically used prescription drugs is from friends or relatives who obtained these medications from medical provider (Garnier-Dykstra et al., 2012, Schepis \& Krishnan-Sarin, 2009), it is likely that adult STM users obtained ADHD stimulants as well as tranquilizers or sedatives from the same source. Further studies of the users' motivations and sources are needed to better understand these distinct substance use profiles in adults and adolescents.

Not surprisingly, respondents in the STM/METH group were more likely to use multiple drugs and to have mental health problems and deviant behaviors compared to STM users; this phenomenon held for adults and adolescents. It indicates that STM/METH group has a higher prevalence of comorbidities and problematic behaviors that require greater clinical attention. Comorbid substance use and psychiatric disorders confer an additional risk for poorer prognosis for individuals with substance use problems (Hasin et al., 2007; Hedden et al., 2009; Strain, 2002). One study pointed out more substance comorbidities indicated not only higher service use but also higher perceived unmet need for treatment (Chen et al., 2013), suggesting that more clinical resources should be allocated to STM/METH group.

This study has several strengths, including its large sample size and generalizability to the US household population. However, our findings should be interpreted in light of several 
limitations, mainly inherent to NSDUH design. First, the cross-sectional survey data limits assessment of temporal relationship. Second, the ascertained methamphetamine users might include medically prescribed Desoxyn or Methedrine users although they were not commonly prescribed (DEA, 2013). Third, among the mental health measures selected in this study, only major depressive episode was assessed via a screening instrument, while ascertainment of anxiety disorder was subject to health care utilization. Fourth, we were not able to explore whether individuals who used ADHD stimulants nonmedically had used these drugs under a provider's supervision in the past, which has implications for prevention strategy. Fifth, NSDUH does not include homeless and jailed individuals who may have a higher prevalence of substance disorders or more severe disorders. Lastly, all the information is based on self-report, which may be vulnerable to recall bias.

\section{Conclusion}

Different population groups are at risk for nonmedical ADHD stimulant use, methamphetamine use or use of both types of drugs. Socio-demographics and psychiatric characteristics, as well as substance comorbidities across the three groups differ in adults and adolescents. Future studies are needed to better explain these differences, including possible differences in the motives for drug use and the temporal relationship of drug use and mental health problems. Given that STM/METH users have more severe psychiatric comorbidities and behavior problems, more clinical and social resources should be allocated to this subgroup.

\section{Acknowledgments}

\section{Role of funding source}

This study was supported by NIDA grants DA023434 (P.I.: Dr. Martins). NIDA had no further role in study design; in the collection, analysis and interpretation of data; in the writing of the report; or in the decision to submit the paper for publication.

\section{References}

Andrews G, Peters L. The psychometric properties of the Composite International Diagnostic Interview. Social Psychiatry and Psychiatric Epidemiology. 1998; 33:80-88. [PubMed: 9503991]

American psychiatric association. Diagnostic and Statistical Manual of Mental Disorders. 4. Washington, DC: 1994.

Arria AM, Caldeira KM, O'Grady KE, Vincent KB, Johnson EP, Wish ED. Nonmedical use of prescription stimulants among college students: associations with attention-deficit-hyperactivity disorder and polydrug use. Pharmacotherapy. 2008; 28:156-169. [PubMed: 18225963]

Brecht ML, O'Brien A, von Mayrhauser C, Anglin MD. Methamphetamine use behaviors and gender differences. Addictive Behaviors. 2004; 29:89-106. [PubMed: 14667423]

Chen LY, Crum RM, Martins SS, Kaufmann CN, Strain EC, Mojtabai R. Service use and barriers to mental health care among adults with major depression and comorbid substance dependence. Psychiatric Services. 2013; 64:863-870. [PubMed: 23728427]

Cody JT. Precursor medications as a source of methamphetamine and/or amphetamine positive drug testing results. Journal of Occupational and Environmental Medicine. 2002; 44:435-450. [PubMed: 12024689]

Darke S, Kaye S, McKetin R, Duflou J. Major physical and psychological harms of methamphetamine use. Drug and Alcohol Review. 2008; 27:253-262. [PubMed: 18368606] 
Drug Enforcement Administration. Department of Justice, Drug Enforcement Administration ARCOS 2: Report 1, Retail drug distribution. 2003.

Drug Enforcement Administration. Methamphetamine. Drug \& Chemical Evaluation Section, Office of Diversion Control, Drug Enforcement Administration; 2013. Retreived on Oct 8, 2013, at http:// www.deadiversion.usdoj.gov/drug_chem_info/meth.pdf

Durell TM, Kroutil LA, Crits-Christoph P, Barchha N, Van Brunt DL. Prevalence of nonmedical methamphetamine use in the United States. Substance Abuse Treatment, Prevention and Policy. 2008; $3: 19$.

Einhorn, C.; Huang, J.; Lavallee, M. The New York Times. New York: 2012. In Their Own Words: Study Drugs'. Retreived on Sep 8, 2013, at http://www.nytimes.com/interactive/2012/06/10/ education/stimulants-student-voices.html?_r=1\&

Ennis, SR.; Ríos-Vargas, M.; Albert, NA. The Hispanic Population: 2010. U.S. Census bureau, Department of Commerce, Economics and Statistics Administration; 2011.

Findling RL, Short EJ, Manos MJ. Short-term cardiovascular effects of methylphenidate and adderall. Journal of the American Academy of Child Adolescent Psychiatry. 2001; 40:525-529. [PubMed: 11349696]

Frosch D, Shoptaw S, Huber A, Rawson RA, Ling W. Sexual HIV risk among gay and bisexual male methamphetamine abusers. Journal of Substance Abuse Treatment. 1996; 13:483-486. [PubMed: 9219145]

Furr CD, Delva J, Anthony JC. The suspected association between methamphetamine ('ice') smoking and frequent episodes of alcohol intoxication: data from the 1993 National Household Survey on Drug Abuse. Drug and Alcohol Dependence. 2000; 59:89-93. [PubMed: 10706978]

Garnier-Dykstra LM, Caldeira KM, Vincent KB, O’Grady KE, Arria AM. Nonmedical use of prescription stimulants during college: four-year trends in exposure opportunity, use, motives, and sources. Journal of American College Health. 2012; 60:226-234. [PubMed: 22420700]

Gonzales R, Marinelli-Casey P, Shoptaw S, Ang A, Rawson RA. Hepatitis C virus infection among methamphetamine-dependent individuals in outpatient treatment. Journal of Substance Abuse Treatment. 2006; 31:195-202. [PubMed: 16919748]

Gruenewald PJ, Johnson FW, Ponicki WR, Remer LG, Lascala EA. Assessing correlates of the growth and extent of methamphetamine abuse and dependence in California. Substance Use and Misuse. 2010; 45:1948-1970. [PubMed: 20380553]

Hasin DS, Keyes KM, Hatzenbuehler ML, Aharonovich EA, Alderson D. Alcohol consumption and posttraumatic stress after exposure to terrorism: effects of proximity, loss, and psychiatric history. American Journal of Public Health. 2007; 97:2268-2275. [PubMed: 17971553]

Hedden SL, Malcolm RJ, Latimer WW. Differences between adult non-drug users versus alcohol, cocaine and concurrent alcohol and cocaine problem users. Addictive Behaviors. 2009; 34:323326. [PubMed: 19059733]

Herman-Stahl MA, Krebs CP, Kroutil LA, Heller DC. Risk and protective factors for nonmedical use of prescription stimulants and methamphetamine among adolescents. Journal of Adolescent Health. 2006; 39:374-380. [PubMed: 16919799]

Herman-Stahl MA, Krebs CP, Kroutil LA, Heller DC. Risk and protective factors for methamphetamine use and nonmedical use of prescription stimulants among young adults aged 18 to 25. Addictive Behavaiors. 2007; 32:1003-1015.

Johnston, LD.; O’Malley, PM.; Bachman, JG. Monitoring the Future National Survey Results on Drug Use, 1975-2002: II. College Students and Adults Ages 19-40. US Department of Health and Human Services; Washington, DC: 2003. NIH publication no. 03-5376

Kaye S, Darke S, Duflou J, McKetin R. Methamphetamine-related fatalities in Australia: demographics, circumstances, toxicology and major organ pathology. Addiction. 2008; 103:13531360. [PubMed: 18855825]

Kessler RC, Berglund P, Demler O, Jin R, Koretz D, Merikangas KR, Rush AJ, Walters EE, Wang PS. The epidemiology of major depressive disorder: results from the National Comorbidity Survey Replication (NCS-R). Journal of American Medical Association. 2003; 289:3095-3105. 
Kroutil LA, Van Brunt DL, Herman-Stahl MA, Heller DC, Bray RM, Penne MA. Nonmedical use of prescription stimulants in the United States. Drug and Alcohol Dependence. 2006; 84:135-143. [PubMed: 16480836]

Martins SS, Mazzotti G, Chilcoat HD. Recent-onset ecstasy use: association with deviant behaviors and psychiatric comorbidity. Experimental and Clinical Psychopharmacology. 2006; 14:275-286. [PubMed: 16893270]

Martins SS, Storr CL, Zhu H, Chilcoat HD. Correlates of extramedical use of OxyContin versus other analgesic opioids among the US general population. Drug and Alcohol Dependence. 2009; 99:5867. [PubMed: 18706774]

Maxwell JC, Brecht ML. Methamphetamine: here we go again? Addictive Behaviors. 2011; 36:11681173. [PubMed: 21875772]

McCabe SE, Boyd CJ, Young A. Medical and nonmedical use of prescription drugs among secondary school students. Journal of Adolescent Health. 2007; 40:76-83. [PubMed: 17185209]

McCabe SE, Knight JR, Teter CJ, Wechsler H. Non-medical use of prescription stimulants among US college students: prevalence and correlates from a national survey. Addiction. 2005; 100:96-106. [PubMed: 15598197]

McCabe SE, Teter CJ. Drug use related problems among nonmedical users of prescription stimulants: a web-based survey of college students from a Midwestern university. Drug and Alcohol Dependence. 2007; 91:69-76. [PubMed: 17624690]

McCabe SE, Teter CJ, Boyd CJ. The use, misuse and diversion of prescription stimulants among middle and high school students. Substance Use and Misuse. 2004; 39:1095-1116. [PubMed: 15387205]

Newton TF, De La Garza R 2nd, Kalechstein AD, Nestor L. Cocaine and methamphetamine produce different patterns of subjective and cardiovascular effects. Pharmacology Biochemistry and Behavior. 2005; 82:90-97.

National Institute on Drug Abuse. DrugFacts: Stimulant ADHD Medications - Methylphenidate and Amphetamines. 2009. Retreived on Oct 8, 2013 at http://www.drugabuse.gov/publications/ drugfacts/stimulant-adhd-medications-methylphenidate-amphetamines

Safer DJ, Zito JM, Fine EM. Increased methylphenidate usage for attention deficit disorder in the 1990s. Pediatrics. 1996; 98:1084-1088. [PubMed: 8951257]

Substance Abuse and Mental Health Services Administration. Methamphetamine Use, Abuse, and Dependence 2002, 2003 and 2004. Substance Abuse and Mental Health Services Administration, Office of Applied Studies; 2005.

Substance Abuse and Mental Health Services Administration. Results from the 2007 National Survey on Drug Use and Health: National Findings. Office of Applied Studies; Rockville, MD: 2008. NSDUH Series H-34, DHHS Publication No. SMA 08-4343

Substance Abuse and Mental Health Services Administration. The NSDUH Report: Nonmedical Use of Adderall ${ }^{\circledR}$ among Full-Time College Students. Substance Abuse and Mental Health Services Administration, Office of Applied Studies; Rockville, MD: 2009a.

Substance Abuse and Mental Health Services Administration. National Admissions to Substance Abuse Treatment Services. Office of Applied Studies; Rockville, MD: 2009b. Treatment Episode Data Set (TEDS): 1997-2007. DASIS Series S-47, DHHS Publication No. SMA 09-4379

Substance Abuse and Mental Health Services Administration. Results from the 2009 National Survey on Drug Use and Health: Summary of National Findings. Office of Applied Studies; Rockville, MD: 2010. NSDUH series H-38A, HHS Publication No. SMA 10-4856

Substance Abuse and Mental Health Services Administration. Results from the 2010 National Survey on Drug Use and Health: Summary of National Findings. Office of Applied Studies; Rockville, MD: 2011. NSDUH series 11-4658, HHS Publication No. SMA 10-4856

Substance Abuse and Mental Health Services Administration. Results from the 2011 National Survey on Drug Use and Health: Summary of National Findings. Office of Applied Studies; Rockville, MD: 2012. NSDUH Series H-44, HHS Publication No. SMA 12- 4713

Schepis TS, Krishnan-Sarin S. Sources of prescriptions for misuse by adolescents: differences in sex, ethnicity, and severity of misuse in a population-based study. Journal of American Academy of Child and Adolescent Psychiatry. 2009; 48:828-836. 
StataCorp. Stata Statistical Software: Release 13. College Station, TX: StataCorp LP; 2013.

Strain EC. Assessment and treatment of comorbid psychiatric disorders in opioid-dependent patients. The Clinical Journal of Pain. 2002; 18:S14-27. [PubMed: 12479251]

Teter CJ, McCabe SE, Cranford JA, Boyd CJ, Guthrie SK. Prevalence and motives for illicit use of prescription stimulants in an undergraduate student sample. Journal of American College Health. 2005; 53:253-262. [PubMed: 15900989]

Teter CJ, McCabe SE, LaGrange K, Cranford JA, Boyd CJ. Illicit use of specific prescription stimulants among college students: prevalence, motives, and routes of administration. Pharmacotherapy. 2006; 26:1501-1510. [PubMed: 16999660]

White BP, Becker-Blease KA, Grace-Bishop K. Stimulant medication use, misuse, and abuse in an undergraduate and graduate student sample. Journal of American College Health. 2006; 54:261268. [PubMed: 16539218]

Wittchen HU. Reliability and validity studies of the WHO--Composite International Diagnostic Interview (CIDI): a critical review. Journal of Psychiatric Research. 1994; 28:57-84. [PubMed: 8064641]

Wu LT, Pilowsky DJ, Schlenger WE, Galvin DM. Misuse of methamphetamine and prescription stimulants among youths and young adults in the community. Drug and Alcohol Dependence. 2007; 89:195-205. [PubMed: 17257780]

Yu Q, Larson DF, Watson RR. Heart disease, methamphetamine and AIDS. Life Science. 2003; 73:129-140.

Zweben JE, Cohen JB, Christian D, Galloway GP, Salinardi M, Parent D, Iguchi M. Psychiatric symptoms in methamphetamine users. American Journal on Addictions. 2004; 13:181-190. [PubMed: 15204668] 


\section{Highlights}

- Methamphetamine and nonmedical ADHD stimulant users have different characteristics.

- Social and mental health profiles of users of different stimulants vary by age.

- Users of both stimulant group have poorer social and mental health outcomes. 


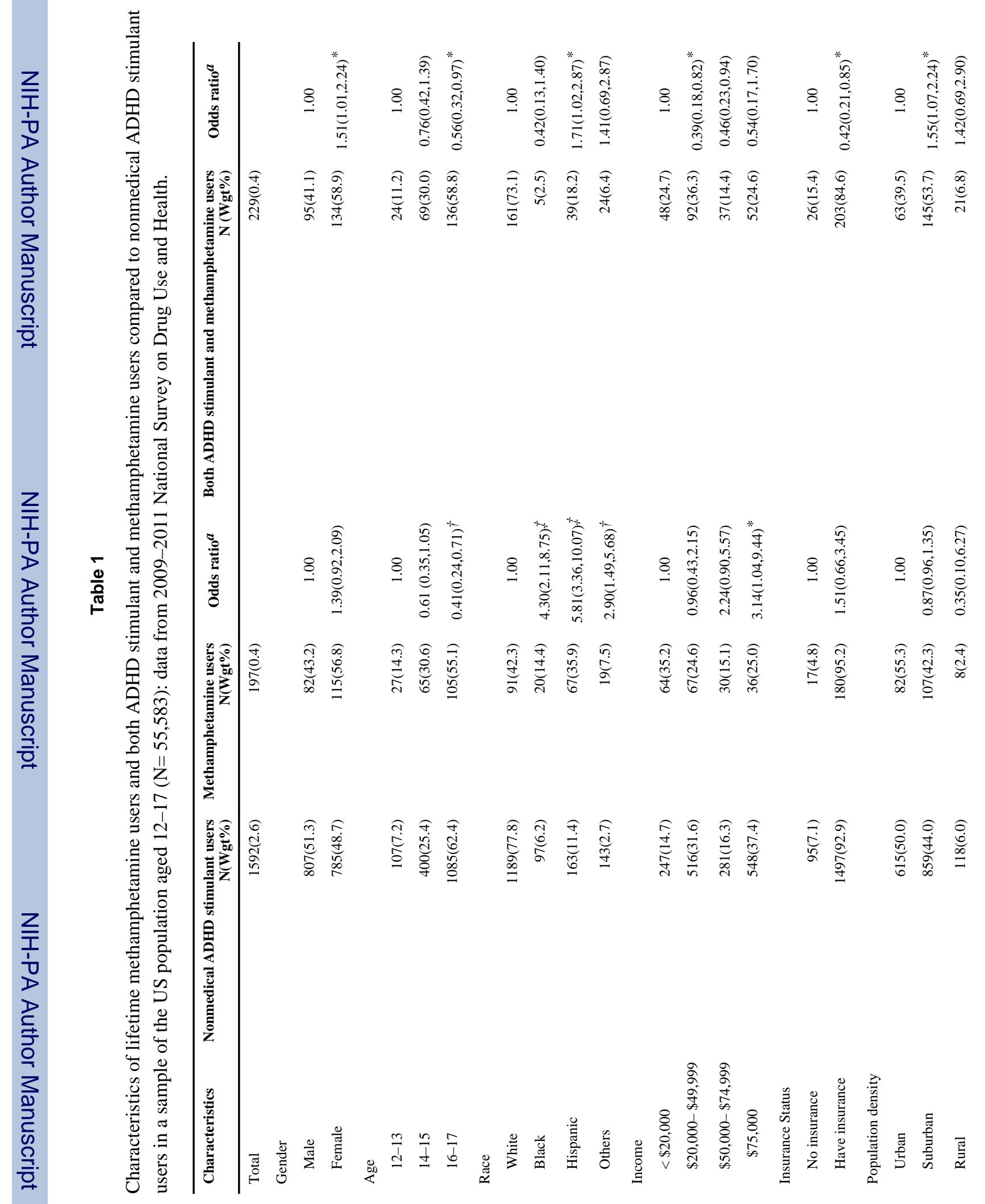




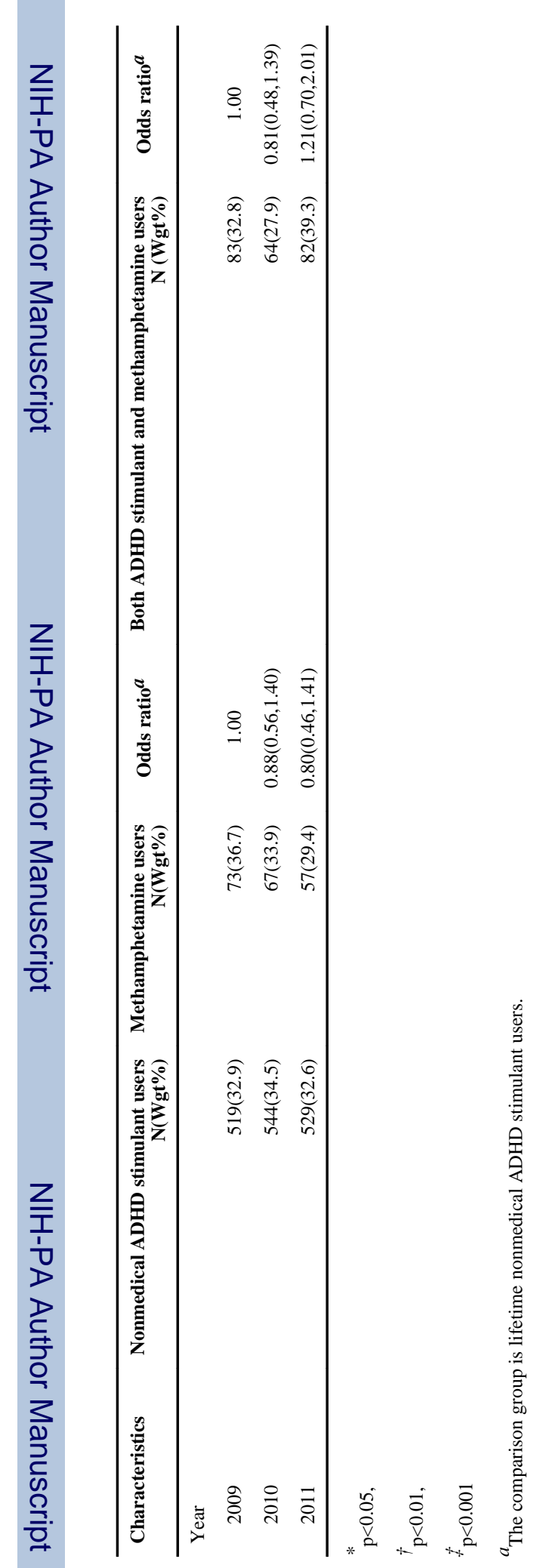

Addict Behav. Author manuscript; available in PMC 2015 May 01. 


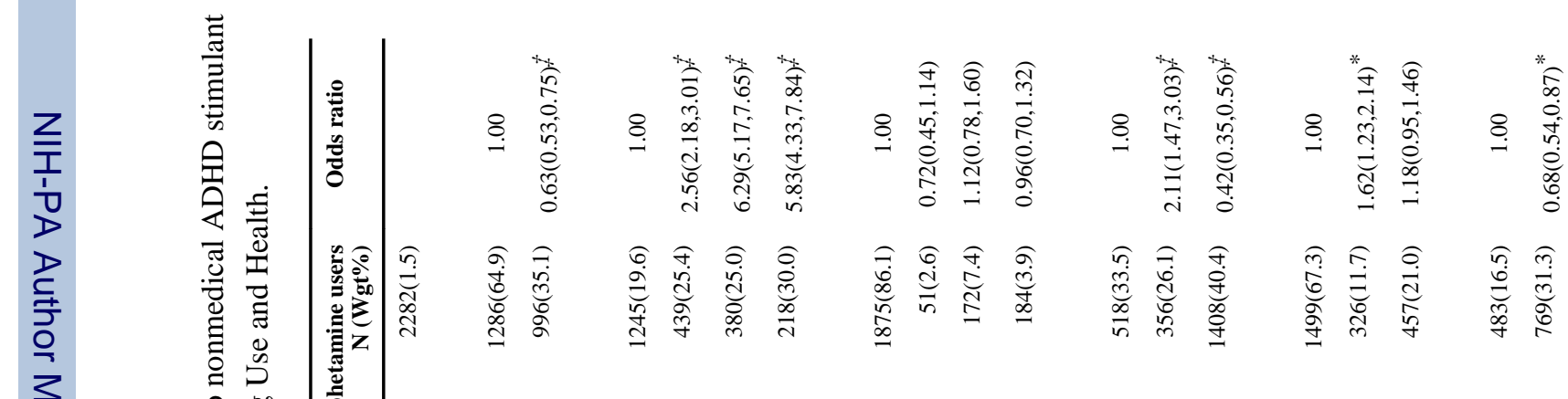

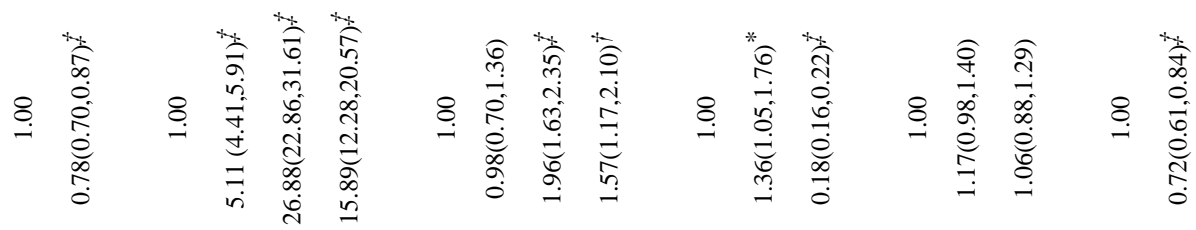

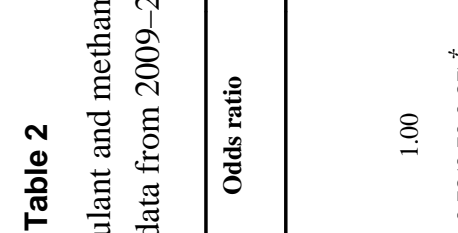

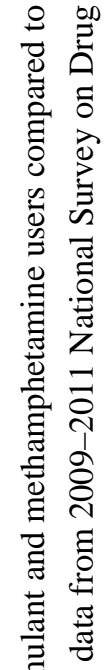
苟 


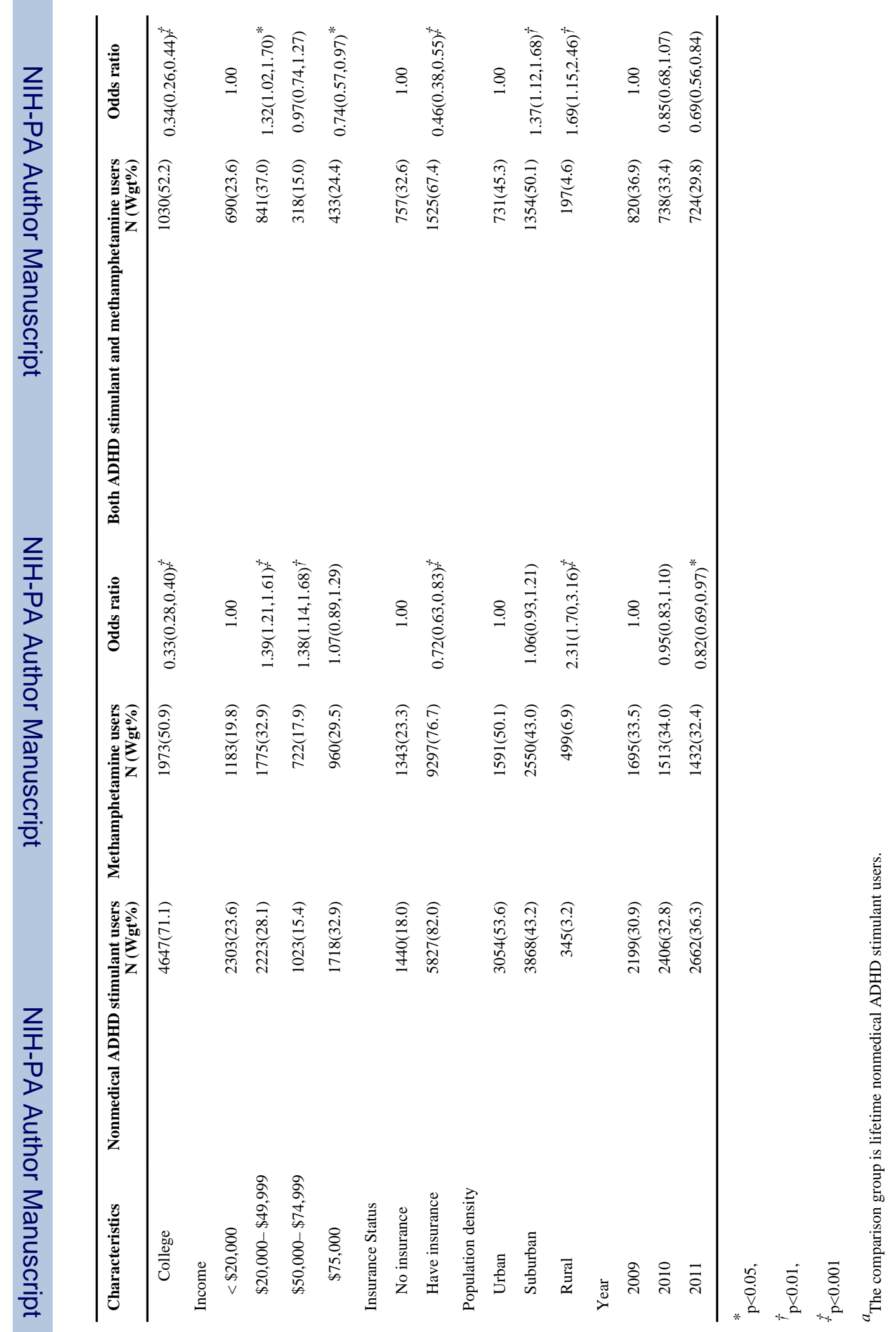




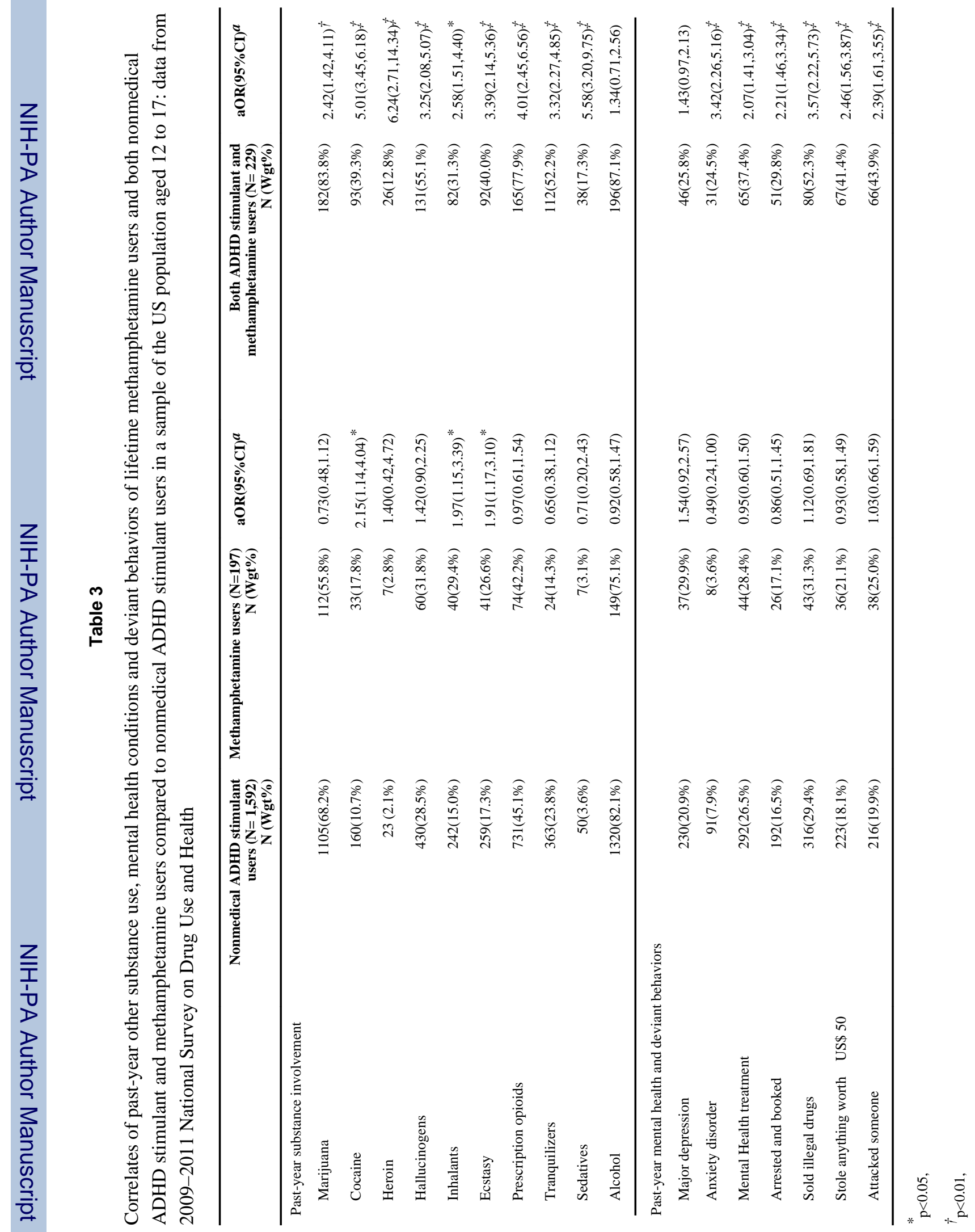

Addict Behav. Author manuscript; available in PMC 2015 May 01. 


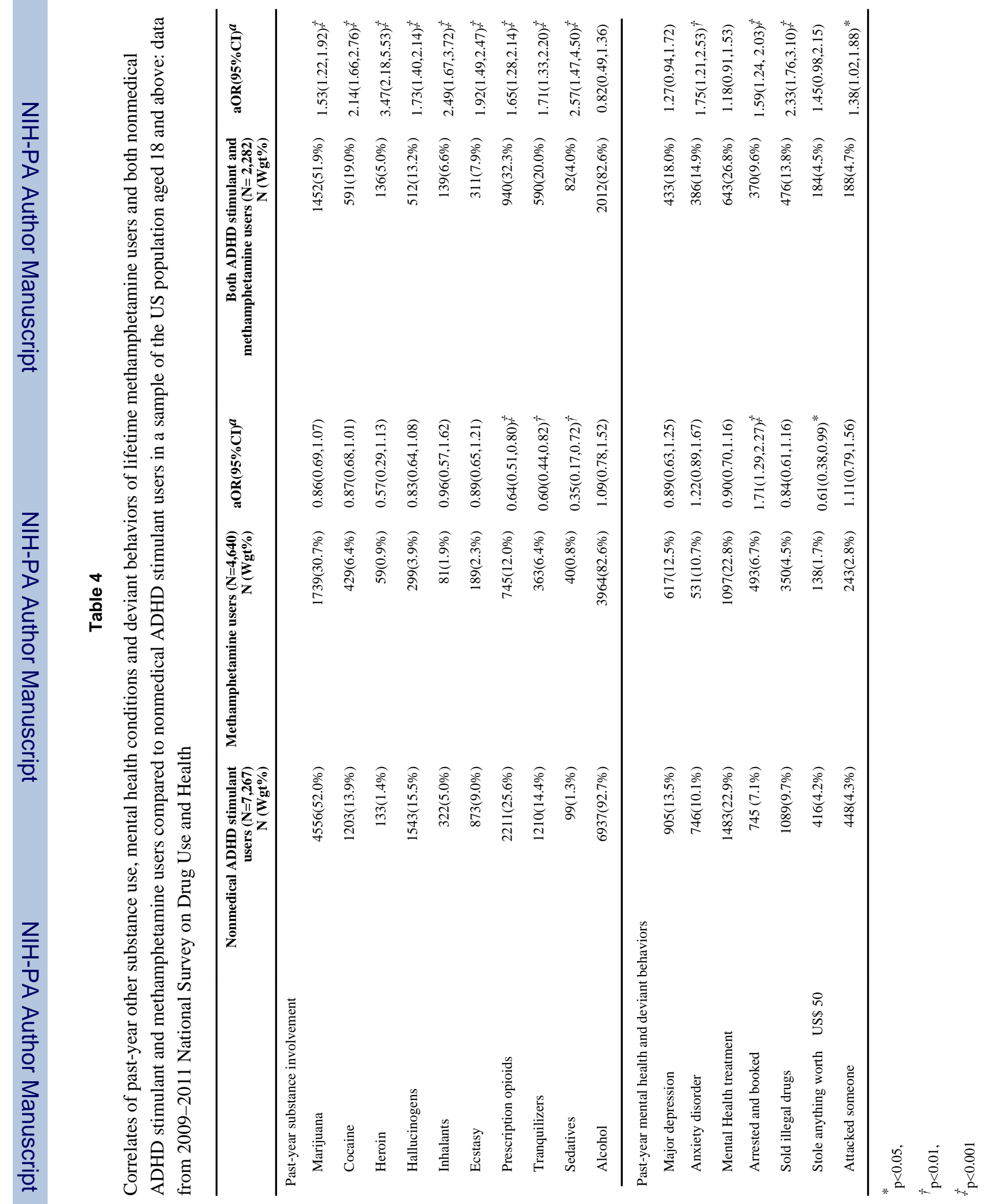


